\title{
A novel gene RNF138 expressed in human gliomas and its function in the glioma cell line $\mathrm{U} 251$
}

\author{
You-xin Zhou ${ }^{\mathrm{a}}$, San-song Chen ${ }^{\mathrm{a}, *}$, Ting-feng Wu ${ }^{\mathrm{a}}$, Da-dong Ding ${ }^{\mathrm{a}}$, Xiong-hui Chen ${ }^{\mathrm{a}}$, Jin-ming Chen ${ }^{\mathrm{a}}$, \\ Zuo-peng $\mathrm{Su}^{\mathrm{a}}$, Bin $\mathrm{Li}^{\mathrm{b}}$, Gui-lin $\mathrm{Chen}^{\mathrm{b}}$, Xue-shun $\mathrm{Xie}^{\mathrm{b}}$, Yan-fang Dai ${ }^{\mathrm{b}}$, Yong-xin Wei ${ }^{\mathrm{b}}$ and $\mathrm{Zi}$-wei $\mathrm{Du}^{\mathrm{b}}$ \\ ${ }^{a}$ Department of Neurosurgery, The First Affiliated Hospital of Soochow University, Suzhou, Jiangsu Province, China \\ ${ }^{\mathrm{b}}$ The Brain and Nerve Research Laboratory, The First Affiliated Hospital of Soochow University, Suzhou, Jiangsu \\ Province, China
}

Received: August 8, 2011

Accepted: October 25, 2011

\begin{abstract}
Background: The gliomas represent the most common primary malignant brain tumors; however, little is known about the molecular pathogenesis of these tumors. Recent research reveals that the oncogenesis and development of gliomas have a close relation to the overexpression of several oncogenes and the inactivation of tumor suppressor genes. Whether the RING finger protein, RNF138, a newly discovered protein, plays a role in glioma oncogenesis is unknown. The present study investigates the expression levels of RNF138 mRNA in glioma samples and noncancerous brain samples and its function in the human glioma cell line U251.

Methods: RT-PCR was used to ascertain the expression of RNF138 mRNA in the glioma cell lines U251, SHG44, U87, A172, and U373. The RNF138 mRNA expression levels of 35 pathological confirmed glioma samples (Grade I - 4 cases, Grade II - 13 cases, Grade III - 11 cases, and Grade IV - 7 cases) and five noncancerous brain tissue samples were analyzed by real-time quantitative PCR. By RNA interference (RNAi) with the lentivirus vector system, the expression of RNF138 was inhibited in the human astrocytomas-glioblastoma multiforme cell line U251. The effects of RNF138-knockdown on cell proliferation were assessed by Cellomics, and cell cycle and cell apoptosis were assessed by FACS.

Results: The RNF138 mRNA is expressed in the five glioma cell lines, and its expression level is significantly higher in glioma tissue than in noncancerous brain tissue. By down-regulation of RNF138 expression, U251 cell proliferation was inhibited and cell apoptosis increased. At the same time, S stage cells lessened and G2 stage cells increased.

Conclusion: The RNF138 gene is highly expressed in glioma tissue and glioma cell lines. It plays an important role in glioma cell proliferation, apoptosis, and cell cycle.
\end{abstract}

Keywords: RNF138, U251, RNA interference, proliferation, apoptosis

\section{Introduction}

Gliomas are one of the most lethal malignancies of the human brain. Despite some advances in early detection, most patients are at advanced stages at the

*Corresponding author: San-song Chen, Department of Neurosurgery, The First Affiliated Hospital of Soochow University, 188 Shizi Street, 215006 Suzhou, Jiangsu Province, China. Tel/Fax: +86 512 67781170; E-mail: mansion1006@hotmail.com. time of diagnosis, and their prognosis still remains poor [1, 2]. Recent advances in human genome studies have opened new avenues for the identification of susceptibility genes for gliomas. Studies have revealed that apart from environmental factors, gene alterations play a key role in the oncogenesis and the progression of the gliomas [2-4]. However, the specific molecular mechanisms remain largely unknown. The identification of putative genes and the characterization of the relationship between changes 
in gene functions and progression of gliomas are urgently needed to isolate potential molecular targets for early diagnosis, future gene therapy, and prognostic indicators.

A RING finger domain is a novel type of zincbinding motif. It is found in a large set of proteins and plays a pivotal role in diverse cellular processes, including oncogenesis, development, signal transduction, and apoptosis that were first identified in the early 1990 s [5]. The RING finger domains can be classified into two subgroups according to the presence of a cysteine or a histidine in the fifth position of the domain: C3HC4 (RING-HC) are mostly nuclear proteins, and the motif is involved in both protein-DNA and protein-protein interactions. The $\mathrm{C} 3 \mathrm{H} 2 \mathrm{C} 3$ (RING$\mathrm{H} 2$ ) variant contains a histidine in place of the fourth cysteine and is able to bind two zinc atoms [5-7]. It has been shown that RING proteins are able to mediate ubiquitin-conjugating enzyme (E2)-dependent ubiquitination in vitro, thus acting as E3 ubiquitin ligases [6]. Various observations showed that genetic alterations in RING finger proteins are implicated in several types of human cancer through different mechanisms, including breast and ovarian cancers, acute promyelocytic leukemia, prostate cancer, and bladder cancer [6, 8-11].

The RING finger protein 138 (RNF138) was first isolated from yeast, two-hybrid termed NLK associated RING finger protein, by Misato Yamada et al in 2006 [12]. It induces the ubiquitylation of TCF/LEF in vitro and in vivo and functions as an E3 ubiquitin-ligase that specifically cooperates with the E2 conjugating enzyme E2-25K. Thus, it regulates the degradation of TCF/LEF and acts as a negative regulator of Wnt signaling. It also inhibits the secondary axis formation induced by ectopic expression of $\beta$-catenin in Xenopus embryos [12]. Nielse reported that the up-regulated RNF138 in Crohn's disease inflamed smokers has a poorer clinical prognosis than nonsmokers with the disease. It may contribute to colonic mucosal regenerative capabilities impaired due to the inhibitory effect of RNF138 on the Wnt/ $\beta$-catenin pathway [13]. Despite these observations, research on the functions of the RNF138 gene is rare. Whether it plays a role in oncogenesis similar to the other RING finger proteins remains a question, particularly in gliomas. In the current study, we first ascertained the elevated expression of RNF138 in the glioma cell lines U251, SHG44, U87, A172, and U373 and glioma samples. We exploited siRNA targeting of RNF138 to down-regulate the expression of RNF138 in the human astrocytoma-glioblastoma multiforme cell line U251 by the lentivirus vector system. Then, we investigated the effects of siRNA on proliferation activity, cell cycle, and cell apoptosis of the U251 cells.

\section{Materials and methods}

\subsection{Cell lines}

The human astrocytoma cell line U251, derived from grade IV astrocytoma-glioblastoma multiforme (GBM), the glioblastoma cell line U87, and the astrocytoma cell line SHG44, were provided by our Brain and Nerve Research Laboratory. Glioblastoma cell line A172 and human glioblastoma-astrocytoma U373 were purchased from the Cell Bank Type Culture Collection of Chinese Academy of Sciences (CBTCCCAS, Shanghai, China). The five cell lines were maintained in Dulbecco's modified Eagle's medium (DMEM, GIBCO) with $10 \%$ fetal bovine serum (FBS, GIBCO) at $37^{\circ} \mathrm{C}$ in a humidified atmosphere of $5 \%$ $\mathrm{CO}_{2}$.

\subsection{Clinical samples}

Before the study began, written informed consent was obtained from all patients who participated and the study was approved by the Ethics Committee of SooChow University. All experiments complied with the current laws of our country. Thirty-five glioma samples were obtained from 35 Chinese patients from March 2009 to Septemper 2010 at the Department of Neurosurgery of The First Affiliated Hospital of Soochow University (Grade I - 4 cases, Grade II - 13 cases, Grade III - 11 cases, and Grade IV - 7 cases, according to the 2007 WHO Classification system). The patients included 19 males and 16 females. The mean age of the patients at the time of surgery was 38 years (male) and 41 years (female). All tumors were obtained from patients with newly diagnosed gliomas who had received no therapy before sample collection. Five adult noncancerous brain tissues were taken from surgical resections of five trauma patients, for whom a partial resection of normal brain tissue was required as decompression treatment to reduce increased intracranial pressure. Permission 
was received from each patient's family. Parts of these surgically removed samples were immediately snap-frozen in liquid nitrogen. The remaining samples were fixed with formalin and embedded in paraffin for histological studies.

\subsection{RNA preparation and semiquantitative RT-PCR analysis}

Total cellular RNAs from the five cell lines were extracted using the Trizol reagent (Invitrogen, USA) according to the protocol of the supplier. Briefly, $2 \mu \mathrm{g}$ total RNA from each sample was reversely transcribed to single-stranded cDNAs according to the manufacturer's protocol (Fermentas, Lithuania). $2 \mu l$ of the cDNA was used as a template for the following PCR. The specific primer pairs are as follow: RNF138 sense:5'-ATGTCCTATTTGTGTGTCTCT TCC-3'; antisense:5'-GCAGTTTGGTATTGGGTTT CTTC- $3^{\prime}$, product size $144 \mathrm{bp}$; the internal control gene is GAPDH and primer pairs are as follows: sense: 5'-CAAGGTCATCCATGACAACTTTG-3'; antisense: $\quad 5^{\prime}$-GTCCACCACCCTGTTGCTGTAG-3, product size 496bp. PCRs were optimized for the number of cycles to ensure product intensity to be within the linear phase of amplification. The PCR protocol consisted of an initial denaturation step at $95^{\circ} \mathrm{C}$ for five minutes, followed by 30 cycles of a three-step program of $95^{\circ} \mathrm{C}$ for 30 seconds, $60^{\circ} \mathrm{C}$ for 30 seconds, $72^{\circ} \mathrm{C}$ for 30 seconds, and a final extension step of $72^{\circ} \mathrm{C}$ for 10 minutes to amplify the RNF138. Products were separated and analyzed on $1.5 \%$ agarose gels.

\subsection{Lentivirus vectors for RNF138 small interfering $R N A$}

Small interfering RNA (siRNA) sequences targeting human RNF138 (GenBank accession number NM_198128) were designed by Genechem Co. Ltd, Shanghai, China, and selected as the template: CTGTAACAGTAATCACCTA. After confirmation by sequencing, the sequences were cloned into the pGCSIL-GFP (GeneChem, Shanghai, China) to generate the lentiviral vectors. The recombinant lentivirus of siRNA targeting RNF138 (RNF138-RNAi-lentivirus) and the negative control lentivirus (Scr- RNAilentivirus) were prepared.

\subsection{Cell culture and lentivirus siRNA gene transfection}

U251 cells in the exponential phase were resuspended in $0.25 \%$ trypsin, then plated in 6-well plates $\left(5 \times 10^{4}\right.$ cells/well $)$ until cell density reached $30 \%$. Then, according to the MOI value (number of lentiviruses: number of cells), the appropriate amounts of lentiviruses were added to the cells. After $24 \mathrm{~h}$ of transfection at $37^{\circ} \mathrm{C}$, the medium was replaced by fresh DMEM medium and incubated for a further $48 \mathrm{~h}$ at $37^{\circ} \mathrm{C}$ in a humidified atmosphere of $5 \% \mathrm{CO}_{2}$.

\subsection{Real-time quantitative RT-PCR}

Total RNAs were extracted from cell cultures after five days of infection using Trizol reagent (Invitrogen USA) according to the manufacturer's instructions. Similarly, the total RNAs of the tumor tissues and control samples were extracted from the frozen tissue. $2 \mu \mathrm{g}$ of total RNA was reverse-transcribed in a $20 \mu \mathrm{l}$ reaction containing 10 units of M-MLV Reverse Transcriptase and $0.5 \mu \mathrm{g}$ of oligo (dT) primer. $2 \mu \mathrm{l}$ of the cDNA was used for real-time PCR. Sequences of realtime PCR primers are described above. The RNF138 and GAPDH genes were amplified with SYBR Master Mixture (Takara, Japan). Results are presented as CT values, defined as the threshold PCR cycle number at which an amplified product is first detected. The average CT was calculated for both RNF138 and GAPDH, and $\triangle \mathrm{CT}$ was determined as the mean of the triplicate CT values for RNF138 minus the mean of the triplicate $\mathrm{CT}$ values for GAPDH. The $2^{-\Delta \Delta^{\mathrm{C}} \mathrm{T}}$ method was used to analyze the relative changes in gene expression.

\subsection{Cell growth assay by the cellomics}

The cells transfected with RNF138-siRNA lentivirus and $\mathrm{NC}$ lentivirus were resuspended in $0.25 \%$ trypsin respectively. The cells then were plated in six-well plates $100 \mu \mathrm{l}$ per well (2000 cells/well dependent on the cell density), respectively, and maintained at $37^{\circ} \mathrm{C}$ in a humidified atmosphere of $5 \%$ $\mathrm{CO}_{2}$. Each group had five wells to equalize the results. After having been plated for one day, cells were counted once a day for five days by the Cellomics method; then, a cell proliferation curve was drawn. 


\subsection{Cell cycle assay by FACS}

Cells infected with RNF138-siRNA lentivirus and $\mathrm{NC}$ lentivirus were plated onto six-well plates in triplicate and incubated at $37^{\circ} \mathrm{C}$ in a humidified atmosphere of $5 \% \mathrm{CO}_{2}$. When the cell density reached $95 \%$, they were collected, washed twice with icecold phosphate-buffered saline (PBS), fixed with 70\% ice-cold ethanol, and then stained with propidium iodide (PI). The analysis of cell cycle distribution was performed by FAC-scan flow cytometer (Becton Dickinson, USA) in accordance with the manufacturer's guidelines.

\subsection{Cell apoptosis assay}

Cells were stained with Allophycocyanin (APC) labeled annexin- $\mathrm{V}$ to discriminate intact cells (annexin $\mathrm{V}-$ ) from apoptotic cells (annexin $\mathrm{V}+$ ). Cells were collected and washed with ice-cold PBS after five-day transfected. A $1 \mathrm{ml}$ binding buffer was added to the collected cells to suspend them (cell density $1 \times 10^{6}$ $\left.-1 \times 10^{7} \mathrm{cell} / \mathrm{ml}\right)$. $100 \mu \mathrm{l}$ cell suspension $\left(1 \times 10^{5}\right.$ $1 \times 10^{6}$ cells) and $5 \mu \mathrm{l}$ annexinV-APC were incubated together for 30 minutes. FACS analysis for annexin- $\mathrm{V}$ was performed by flow cytometer (Becton Dickinson, USA). All experiments were performed in triplicate.

\subsection{Statistical methods}

All values in the text and figures are expressed as the Mean \pm SEM of these observations. Statistical analysis was performed using SPSS software (Release 13.0, SPSS Inc.). The difference between the two groups was analyzed by the Student's $t$-test. A value of $P<0.05$ was considered to be of statistical significance.

\section{Results}

\subsection{The expression of RNF138 mRNA in the 5 glioma cell lines}

To ascertain the expression of RNF138 mRNA in the glioma cell lines, we detected RNF138 mRNA levels in the glioma cell lines U251, SHG44, U87, A172, and U373 by semi-quantitative RT-PCR. RNF138 is expressed in all the five cell lines (Fig. 1).

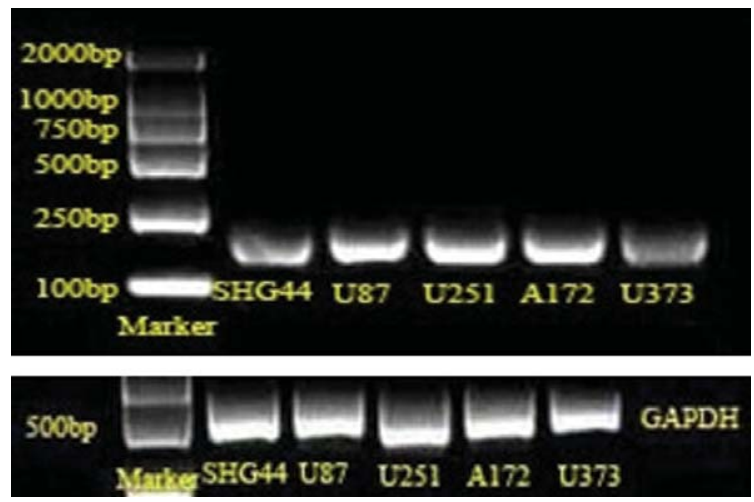

Fig. 1. The expression of RNF138mRNA in the 5 gliomas cell lines by RT-PCR.

\subsection{The relative expression levels of $R N F 138$ $m R N A$ in the tissue samples}

We analyzed RNF138 mRNA expression levels in 35 glioma samples and five noncancerous brain tissue samples to explore whether there was a significant difference between them. The results revealed elevated expression levels of RNF138 in glioma samples compared to the noncancerous brain tissue (Fig. 2a). With the increased level of glioma malignancy, we found that RNF138 mRNA expression increased but that it did not include Grade IV (Fig. 2b).

\subsection{GFP expression of cells transfected with recombinant lentivirus}

A lentivirus vector system with GFP as a reporter gene was used to express siRNA against RNF138. To determine whether the recombinant vectors were efficiently transfected into U251cells, we assessed cells transfected with RNF138-RNAilentivirus (KD), the negative control lentivirus (NC), and the nontransfected. After transfected for $72 \mathrm{~h}$, more than $80 \%$ of U251 cells expressed GFP, indicating a high and stable transfection efficiency. No significant difference was seen between the negative control group and the nontransfected group, indicating that the transfection process had no effect on cell growth (Fig. 3).

\subsection{Silencing of the RNF138 gene by RNA interference}

To verify whether the RNF138 gene was silenced by the vector expressing siRNA, we detected its 

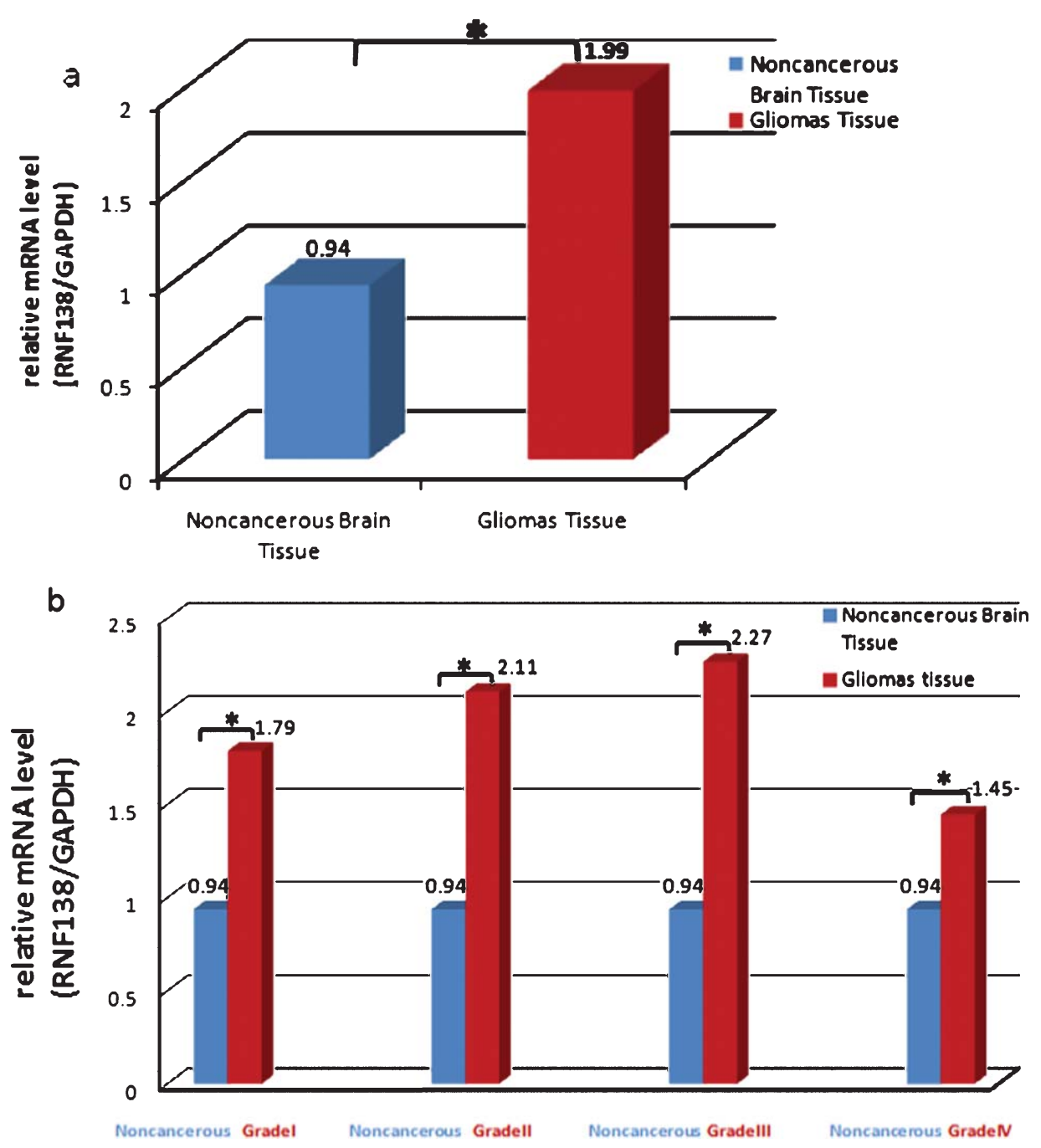

Fig. 2. The relative expression levels of RNF138 mRNA in the gliomas samples and the noncancerous brain tissue detected by real-time quantitative PCR. (a) the elevated expression level of RNF138 in all the 35 gliomas samples (including the Grade I to Grage IV) versus the noncancerous brain tissue. $(0.94 \pm 0.16$ vs. $1.99 \pm 0.36$, respectively; $p=0.01)$. (b) the elevated expression level of each grade gliomas versus the noncancerous brain tissue. $* P<0.05$.

mRNA expression in U251cells transfected with the RNF138-RNAi-lentivirus (KD) and negative control lentivirus (NC) by real-time quantitative PCR. Cells transfected with the RNF138-RNAi showed a significant decrease in the level of mRNA expression relative to the negative control cells $(P<0.05)$ (Table 1 , Fig. 4). The relative mRNA expression levels were analyzed by $2^{-\Delta \Delta C T}$ method $[14,15]$. Thus, RNF138specific RNAi down-regulated RNF138 expression efficiently.

\subsection{Inhibition of cell proliferation by RNAi for RNF 138}

To explore the function of RNF138 expression on cell growth, U251 cells transfected with RNF138siRNA lentivirus and NC lentivirus were counted by Cellomics for five days after the transfection procedure. The cell proliferation ability was inhibited significantly in RNF138-RNAi cells compared to the negative control cells $(P<0.05)$ (Table 2 , 


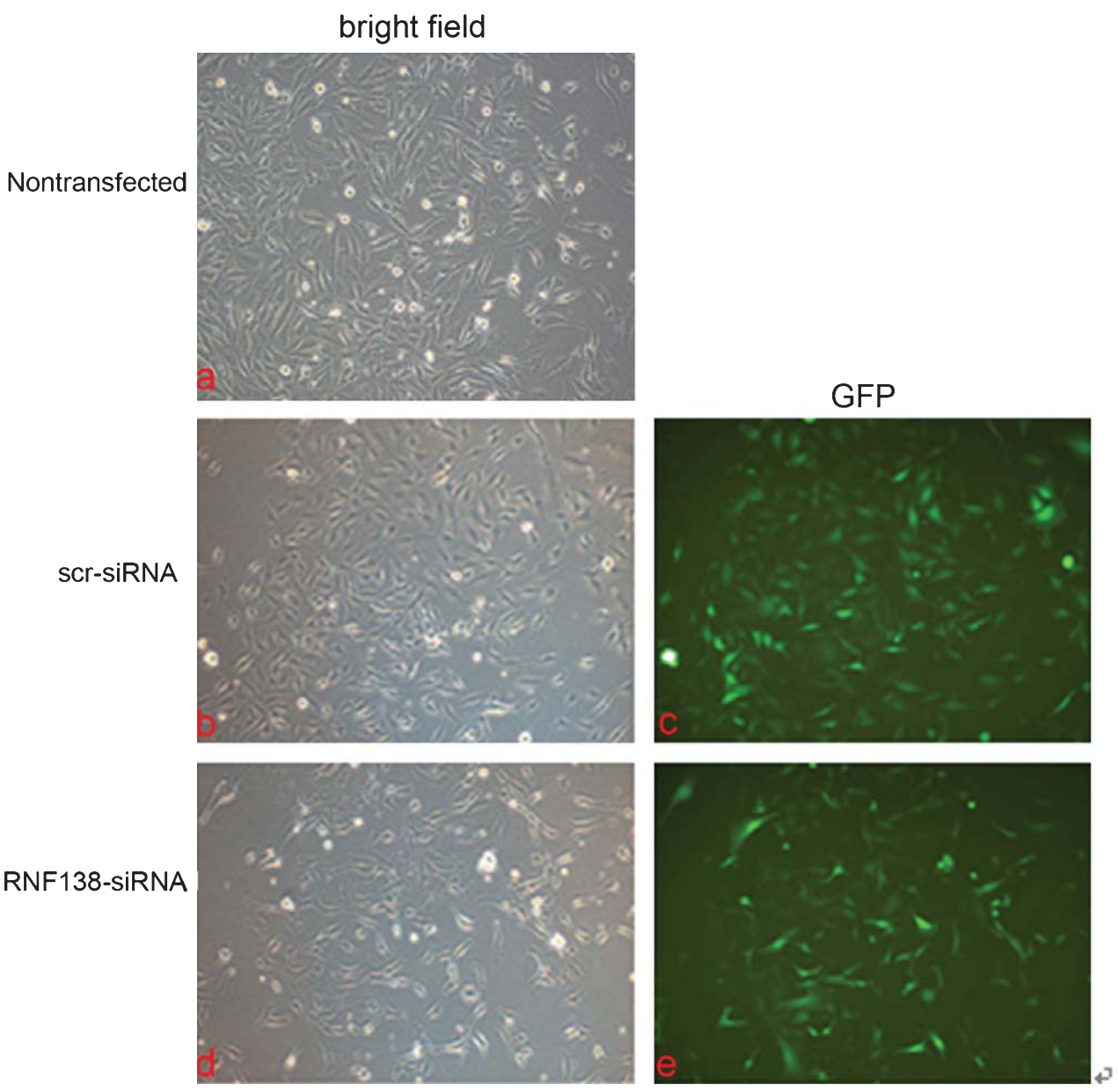

Fig. 3. Cells transfected with RNF138-RNAi-Lentivirus (KD) and negative-control-Lentivirus (NC) and nontransfected were observed under light microscopy (left) and fluorescence microscopy (right) after transfected with the specific lentivirus for $72 \mathrm{~h}$. More than $80 \%$ of U251 cells expressed GFP (right). No significant difference between the negative control group and the nontransfected group, indicating the transfection process has no effect on cells' growth. a: $100 \times \mathrm{B}$; b: NC $100 \times \mathrm{B}$; c: NC $100 \times \mathrm{G}$; d: KD $100 \times \mathrm{B}$; e: KD $100 \times \mathrm{G}$.

Fig. 5). These results indicated that the knockdown of RNF138 expression significantly inhibited the U251 cells growth and might play an important role in cell proliferation.

\subsection{Effect of RNF138-RNAi on cell cycle of U251 cells}

Flow cytometry assay was used to detect whether the cell cycle was affected by RNF138-RNAi. We found that after being transfected with the RNF138-RNAilentivirus, the percentage of $\mathrm{S}$ stage cells was decreased significantly, while the percentage of G2/M stage cells was increased significantly when compared with cells transfected with negative control vector $(P<0.05)$. The percentage of $\mathrm{S}$ stage cells in the group of NC and $\mathrm{KD}$ was $(33.43 \pm 2.20) \%$ and $(26.90 \pm 0.70) \%$, respectively. The percentage of $\mathrm{G} 2 / \mathrm{M}$ stage cells in the group of $\mathrm{NC}$ and $\mathrm{KD}$ was $(15.10 \pm 1.42) \%$ and $(22.89 \pm 0.25) \%$, respectively. The differences of the G1 stage cells were of no statistical significance. These results are shown in Fig. 6.

\subsection{Effect of RNF138-RNAi on cell apoptosis of U251 cells}

Since the RNF138 plays a role in maintaining the cell growth and cell cycle, it is not known whether its 
Table 1

CT values of GAPDH and RNF138 detected by real-time quantitative PCR

\begin{tabular}{lcccc}
\hline Sample & GAPDH CT valve average & RNF138 CT value average & $2^{-\Delta \Delta C T}$ average & $p$ value \\
\hline Scr-siRNA & $15.34 \pm 0.032$ & $25.14 \pm 0.079$ & $1.002 \pm 0.074$ & 0.001 \\
RNF138-siRNA & $15.96 \pm 0.026$ & $27.04 \pm 0.151$ & $0.414 \pm 0.037$ & \\
\hline
\end{tabular}

CT values of GAPDH and RNF138 detected by real-time quantitative PCR and the relative mRNA expression levesl were analyzed by $2^{-\Delta \Delta \mathrm{CT}}$ method.

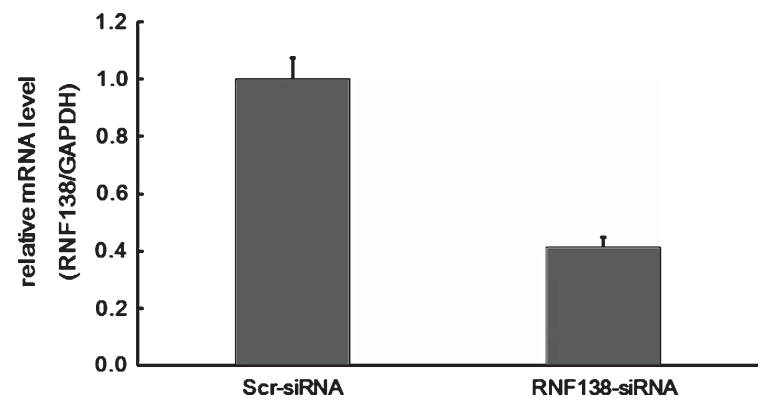

Fig. 4. Bar charts of the the relative mRNA expression levels between the RNF138-RNAi-Lentivirus (KD) and negative control Lentivirus (NC). The RNF138 mRNA level decreased significantly by knocking down method.

Table 2

Cell numbers counted by cellomics

\begin{tabular}{lrrr}
\hline AV/num & \multicolumn{1}{c}{ scr-SiRNA } & RNF138-SiRNA & $p$ value \\
\hline Day1 & $709.40 \pm 76.49$ & $555.2 \pm 36.36$ & 0.03 \\
Day2 & $908.20 \pm 69.44$ & $627.00 \pm 40.77$ & \\
Day3 & $1094.80 \pm 64.57$ & $705.80 \pm 42.02$ & \\
Day4 & $1287.60 \pm 58.34$ & $781.80 \pm 56.06$ & \\
Day5 & $1549.00 \pm 85.31$ & $868.80 \pm 65.34$ & \\
\hline
\end{tabular}

Cell numbers counted for 5 days after transfected with RNF138siRNA lentivirus and NC lentivirus. The difference between two groups was analyzed by the Student's $t$-test. The $p$ value $=0.03$.

inhibition will lead to apoptosis. We found that after being transfected with RNF138-RNAi-lentivirus for five days, cell apoptosis increased significantly compared to the negative control. The results are shown in Fig. 7.

\section{Discussion}

RING finger protein, which has RING motifs containing the consensus sequence $\mathrm{CX} 2 \mathrm{CX}(9$ -39)CX(1-3)HX(2-3)C/HX2CX(4-48)CX2 C with the Cys and His representing zinc binding residues, have been known for two decades [7, 16]. RING finger proteins such as E3-ubiquitin ligases mediated ubiquitin-conjugating enzyme (E2)-dependent ubiquitination for targeted proteins. Subsequently, the targeted proteins were degraded [17]. It is becoming more apparent that ubiquitin-mediated proteolysis is responsible for the regulation of the levels of a wide variety of cellular proteins. The deregulation of ubiquitin-mediated proteolysis can be detrimental to cellular homeostasis. The resultant loss of protein function can lead to cellular imbalances and tumorigenesis. Research has revealed an association between the expression of components of the ubiquitin proteolysis pathway and cancer progression. Increased levels of E3-ligases have been observed in numerous different tumor types [18-20]. In addition, they are involved in biological processes such as signal transduction, transcriptional regulation, ubiquitination, and apoptosis [16, 21, 22]. The RNF138 protein, which first was isolated using the yeast two-hybrid method, functioned as an E3 ubiquitin-ligase to induce the ubiquitylation of TCF/LEF. Misato Yamada et al found that microinjection of RNF138 mRNA inhibited secondary axis formation in Xenopus embryos [12]. Nielsen et al found that up-regulated RNF138 in Crohn's disease inflamed smokers had a poorer clinical prognosis than non-smokers with $\mathrm{CD}$. It may contribute to colonic mucosal regenerative capabilities impaired due to the inhibitory effect of RNF138 [13]. These results indicate that RNF138 may correlate to embryo development, cell proliferation, and cell regeneration. Interestingly, in previous research, through high-throughput gene screening technology, we found that the RNF138 gene is expressed in glioma cell lines. The following questions remain to be answered: whether it plays a role as an oncogene similar to other RING finger protein genes; whether it is up-regulated in samples from noncancerous brain tissue; whether it initiates tumors when losing control of the balance between proliferation and apoptosis; 

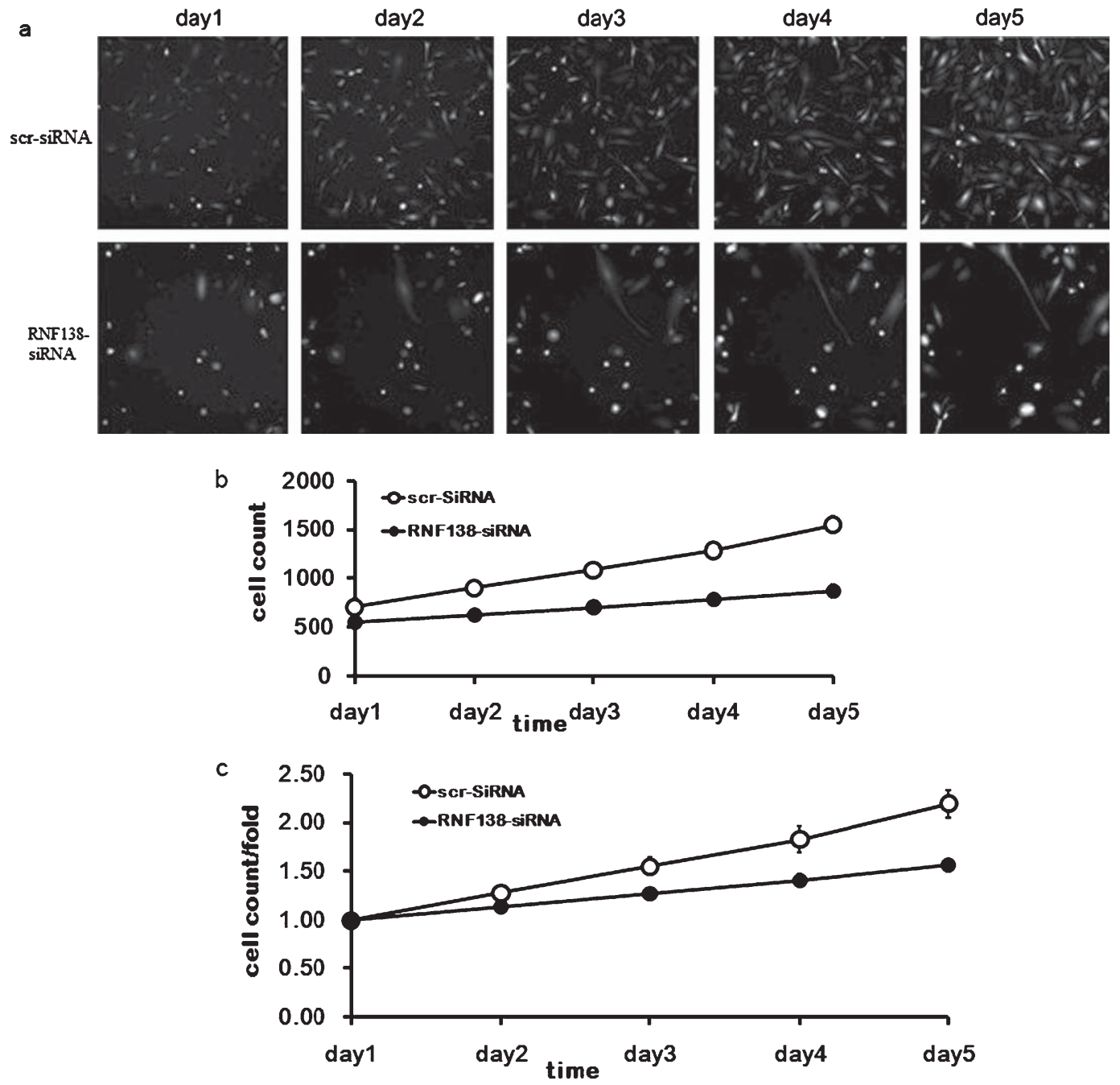

Fig. 5. Effect of down-regulation of RNF138 on cell proliferation: (a) RNF138-siRNA targeted cells and negative control cells were observed by cellomics for 5 days. The cell proliferation ability was significantly inhibited in RNF138-RNAi cells as compared with that negative control cells. (b) cell count curve of RNF138-siRNA targeted cells and negative control cells. (c) cell count/fold curve of RNF138-siRNA targeted cells and negative control cells. These results indicate that knockdown of RNF138 expression significantly inhibits the U251 cells growth.

and whether it is crucial to maintaining glioma cells proliferation and controlling cell apoptosis.

Centering on these questions, in the present study, we found that RNF138 was expressed in the five glioma cell lines by RT-PCR. Next, we examined 35 pathological confirmed gliomas samples and five noncancerous brain tissue by real-time quantitative PCR. The results revealed elevated expression levels of RNF138 in glioma samples compared to noncancerous brain tissue. With the increase of glioma malignancy levels, the RNF138 mRNA expression increased but did not include Grade IV. This may be attributed to necrosis in the Grade IV samples, which had a poor corresponding blood supply adapted to the rapid growth of the tumors. To make clear its functions on cell line, for the first time, we designed and synthesized an RNF138-siRNA expression vector and a negative control vector, which were transfected stably into the U251 human astrocytoma-glioblastoma multiforme cell line. The expression of RNF138 mRNA was significantly 

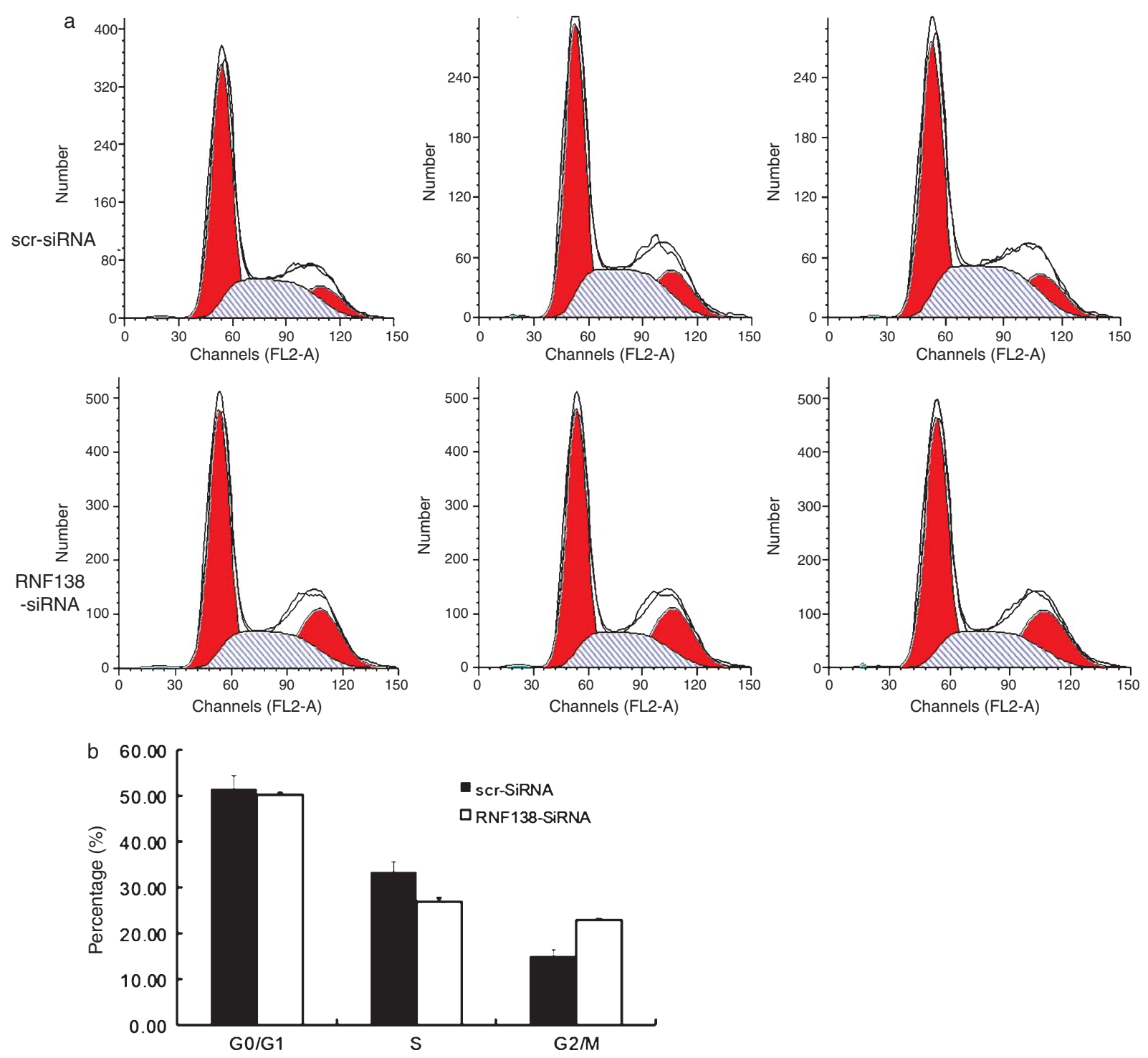

Fig. 6. Effect of down-regulation of RNF138 on cell cycle of U251 cells. (a) Cell cycle analysis by flow cytometry: S stage cells was significantly decreased, while the G2/M stage cells was significantly increased when compared to the negative control cells $(P<0.05)$. (b) bar charts of cell cycle was drawn according to result of the FACS. The results of cell cycle examination demonstrated that the number of cells in G2/M phase was significantly increased in RNF138-siRNA targeted cell groups ( $p=0.009$, compared with negative control), while S stage cells was significantly decreased $(p=0.03)$. No change in the percentage of cells in G1 phase was seen.

inhibited in U251-RNAi cells compared to the negative control cells. The stably transfected cells with RNF138 silencing showed that the proliferation rate decreased, the apoptotic rate increased, and the cell cycle was redistributed. For the results outlined above, we suggest that RNF138 could be an oncogene and that it could be knocked down to decrease the glioma cells proliferation. This leads us to believe that it would be a potential molecular target for early diagnosis and future gene therapy on gliomas. 
The limitation of this research was that the RNF138 functions research was done in vitro. Animal models are needed to confirm our results in vivo in additional research. Moreover, more glioma samples and noncancerous brain samples are necessary to confirm the oncogenic role of RNF138.
This is the first report analyzing RNF138 expression in gliomas and its function in glioma cell lines. However, the specific mechanisms on how the RNF138 influences cell growth and the biological characteristics of the glioma cell line is unclear. Interaction between the RNF138 and Wnt signaling pathway
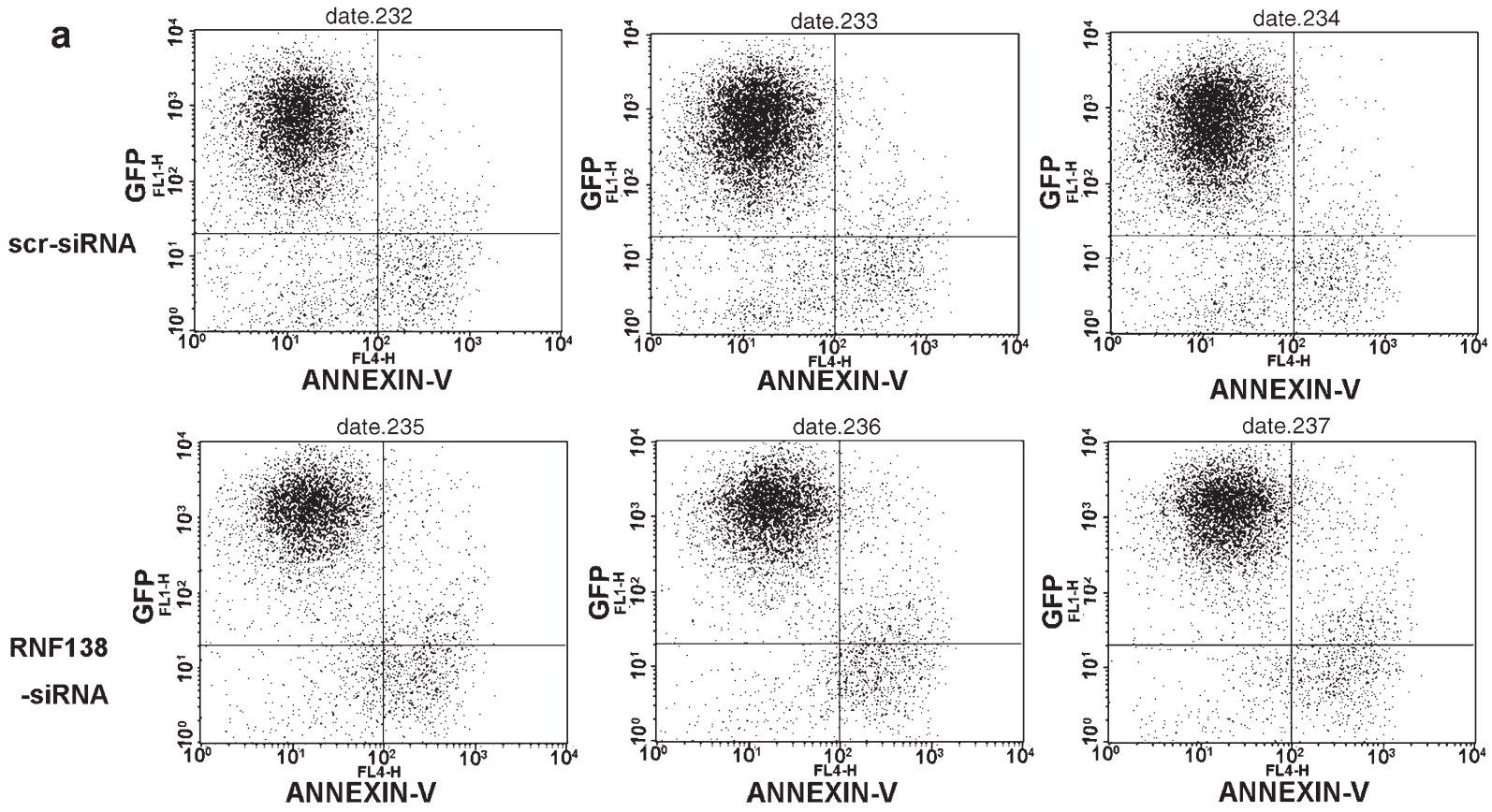

b

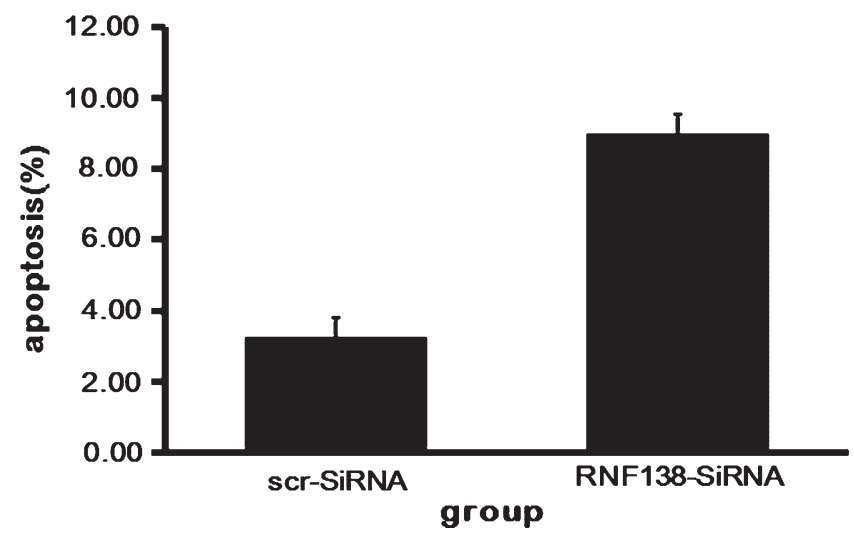

Fig. 7. Effect of down-regulation of RNF138 on cell apoptosis of U251 cell. It was detected after five-day transfected. (a) scatterplot chart of cell apoptosis by FACS. (b) bar charts of cell apoptosis rate was drawn according to result of the FACS. The apoptosis rate in this group of NC and $\mathrm{KD}$ is $(3.26 \pm 0.54) \%,(9.0 \pm 0.55) \%$ respectively $(p=0.0003)$. (c) Apoptosis peak was detected by FACS. (d) bar charts of cell apoptosis rate was drawn. The apoptosis rate in this group of NC and KD is $(9.23 \pm 1.82) \%,(17.16 \pm 0.43) \%$ respectively $(p=0.01)$. The results indicated that down-regulation of RNF138 increased the cell apoptosis of U251 cells. 

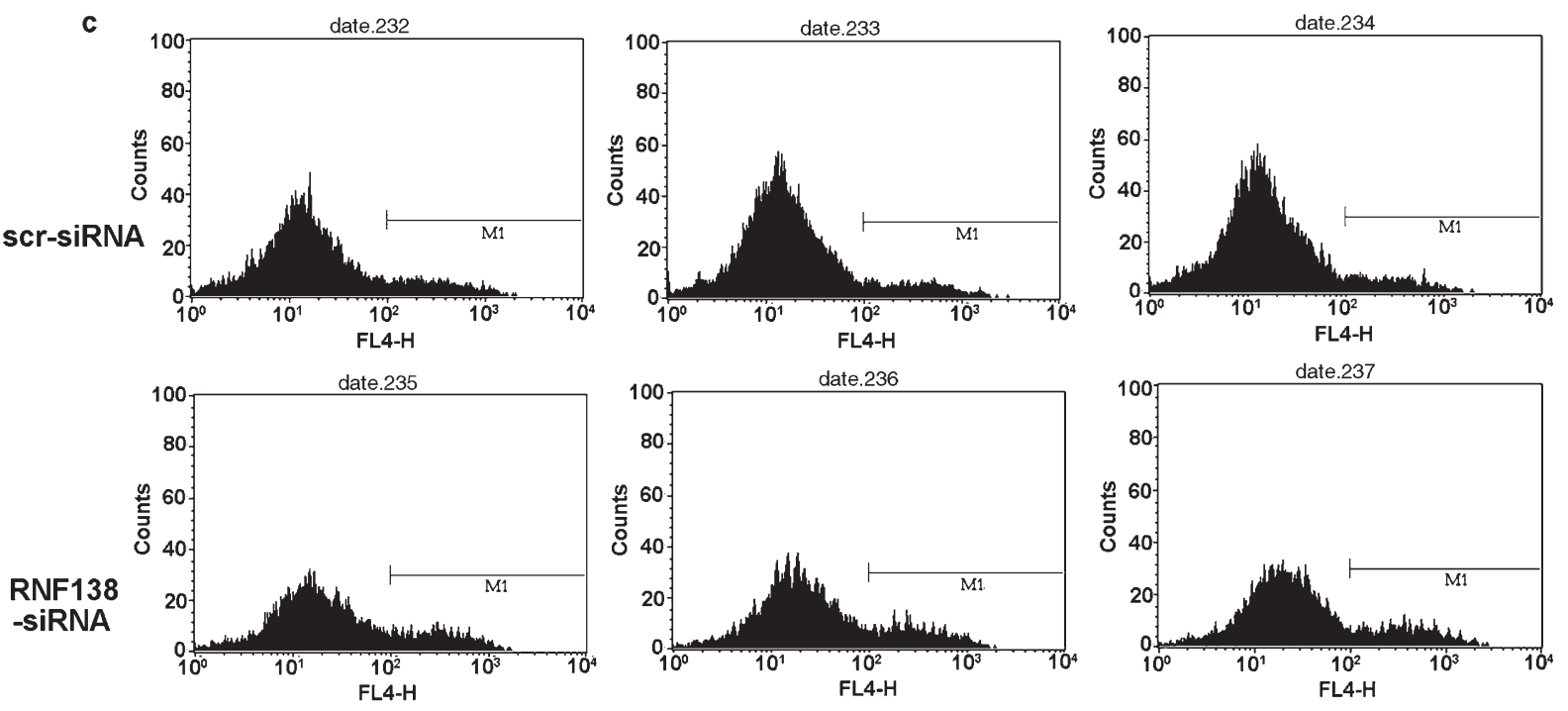

d

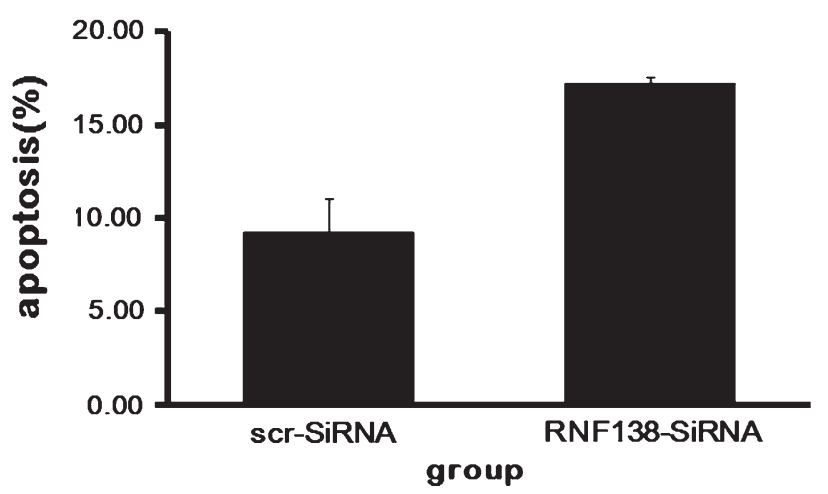

Fig. 7. Continued.

also needs further study which is ongoing in our laboratory.

\section{Acknowledgments}

We thank our patients for their cooperation and Genechem Co. Ltd for providing us with the lentiviral particles and technical assistance. This work was partially supported by major issues Foundation of health department in Jiangsu province (XK 200722, K201106) and Suzhou science and technology plan projects (SYS201025).

The collection of the tumor samples and noncancerous brain tissue was done with the agreement of all of the patients, and written informed consent was obtained from all participants. The study was approved by the Ethics Committee of SooChow University. All of the experiments comply with the current laws of our country.

\section{References}

[1] Y.X. Zhou, F. Liu, Q.N. Xu and X.Y. Wang, Analysis of the expression profile of Dickkopf-1 gene in human glioma and the association with tumor malignancy. J Exp Clin Canc Res 29 (2010), 138

[2] E.A. Maher, F.B. Furnari, R.M. Bachoo, D.H. Rowitch, D.N. Louis, W.K. Cavenee and R.A. DePinho, Malignant glioma: genetics and biology of a grave matter, Genes Dev 15 (2001), 1311-1333. 
[3] Y. Liu, S. Shete, F. Hosking, L. Robertson, R. Houlston and M. Bondy, Genetic advances in glioma: Susceptibility genes and networks, Curr Opin Genet Dev 20 (2001), 239-244.

[4] R.H. Osborne, M.P. Houben, C.C. Tijssen, J.W. Coebergh and C.M van Duijn, The genetic epidemiology of glioma, Neurology 57 (2001), 1751-1755.

[5] T. Sugiura, A. Yamaguchi and K. Miyamoto, A cancerassociated RING finger protein, RNF43, is a ubiquitin ligase that interacts with a nuclear protein, HAP95, Exp Cell Res 314 (2008), 1519-1528.

[6] I. Eisenberg, H. Hochner, T. Levi, R. Yelin, T. Kahan and S. Mitrani-Rosenbaum, Cloning and characterization of a novel human gene RNF38 encoding a conserved putative protein with a RING finger domain, Biochem Biophys Res Commun 294 (2002), 1169-1176.

[7] C.A. Joazeiro and A.M. Weissman, RING finger proteins: mediators of ubiquitin ligase activity, Cell 102 (2000), 549-552.

[8] P.S. Freemont, Ubiquitination: RING for destruction? Curr Biol 10 (2000), 84-87.

[9] K.L. Borden, M.N. Boddy, J. Lally, N.J. O’Reilly, S. Martin, K. Howe, E. Solomon and P.S. Freemont, The solution structure of the RING finger domain from the acute promyelocytic leukaemiaproto-oncoprotein PML, EMBO J 14 (1995), 1532-1541.

[10] I. Fadl-Elmula, L. Gorunova, N. Mandahl, P. Elfving, R. Lundgren, F. Mitelman and S. Heim, Karyotypic characterization of urinary bladder transitional cell carcinomas, Genes Chromosom Canc 3 (2000), 256-265.

[11] G. Perinchery, N. Bukurov, K. Nakajima, J. Chang, L.C. Li and R. Dahiya, High frequency of deletion on chromosome 9p21 may harbor several tumor-suppressor genes in human prostate cancer, Int J Cancer 83 (1999), 610-614.

[12] M. Yamada, J. Ohnishi, B. Ohkawara, S. Iemura and K. Satoh, NARF, an Nemo-like Kinase (NLK)-associated RING Finger
Protein Regulates the Ubiquitylation and Degradation of T Cell Factor/Lymphoid Enhancer Factor (TCF/LEF), J Biol Chem 281 (2006), 20749-20760.

[13] O.H. Nielsen, J.T. Bjerrum, C. Csillag, F.C. Nielsen and J. Olsen, Influence of smoking on colonic gene expression profile in Crohn's disease, PLoS One 4 (2009), e6210.

[14] K.J. Livak and T.D. Schmittgen, Analysis of relative gene expression data using real-time quantitative PCR and the $2^{-\Delta \Delta}$ CT Method, Methods 25 (2001), 402-408.

[15] A. Giulietti, L. Overbergh, D. Valckx, B. Decallonne, R. Bouillon and C. Mathieu, An overview of real-time quantitative PCR: Applications to quantify cytokine gene expression, Methods 25 (2001), 386-401.

[16] K.L. Borden, RING domains: Master builders of molecular scaffolds? J Mol Biol 295 (2000), 1103-1112.

[17] M. Ogawa, K. Mizugishi, A. Ishiguro, Y. Koyabu, Y. Imai, R. Takahashi and K. Mikoshiba, J. Aruga, Rines/RNF180, a novel RING finger gene-encoded product, is a membranebound ubiquitin ligase, Genes Cells 13 (2008), 397-409

[18] K.M. Sakamoto, Ubiquitin-dependent proteolysis: Its role in human diseases and the design of therapeutic strategies, $\mathrm{Mol}$ Genet Metab 77 (2002), 44-56.

[19] M.K. Connor and A. Seth, A central role for the ring finger protein RNF11 in ubiquitin-mediated proteolysis via interactions with E2s and E3s, Oncogene 23 (2004), 2089-2095

[20] H.C. Ardley, Ring finger ubiquitin protein ligases and their implication to the pathogenesis of human diseases, Curr Pharm Des 1 (2009), 3697-715.

[21] D. Guardavaccaro, M. Pagano, Oncogenic aberrations of cullin-dependent ubiquitin ligases, Oncogene 23 (2004), 2037-2049.

[22] C.M. Pickart, Mechanisms underlying ubiquitination, Annu Rev Biochem 70 (2001), 503-533. 


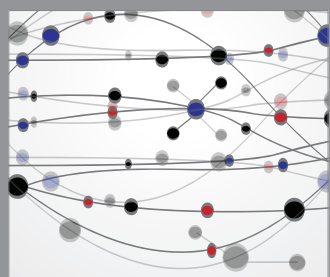

The Scientific World Journal
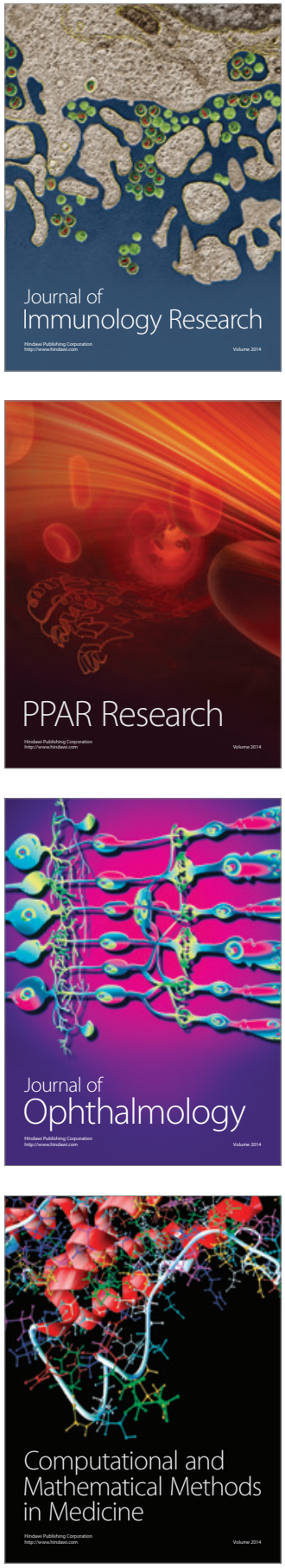

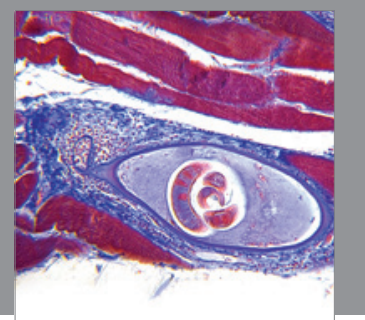

Gastroenterology

Research and Practice
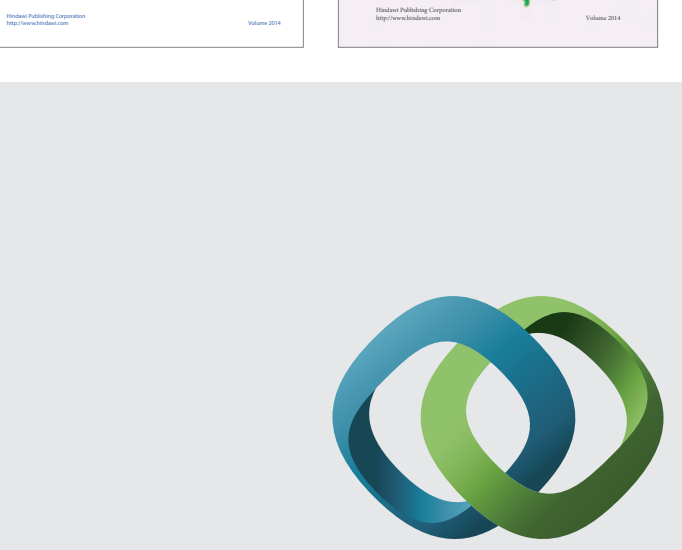

\section{Hindawi}

Submit your manuscripts at

http://www.hindawi.com
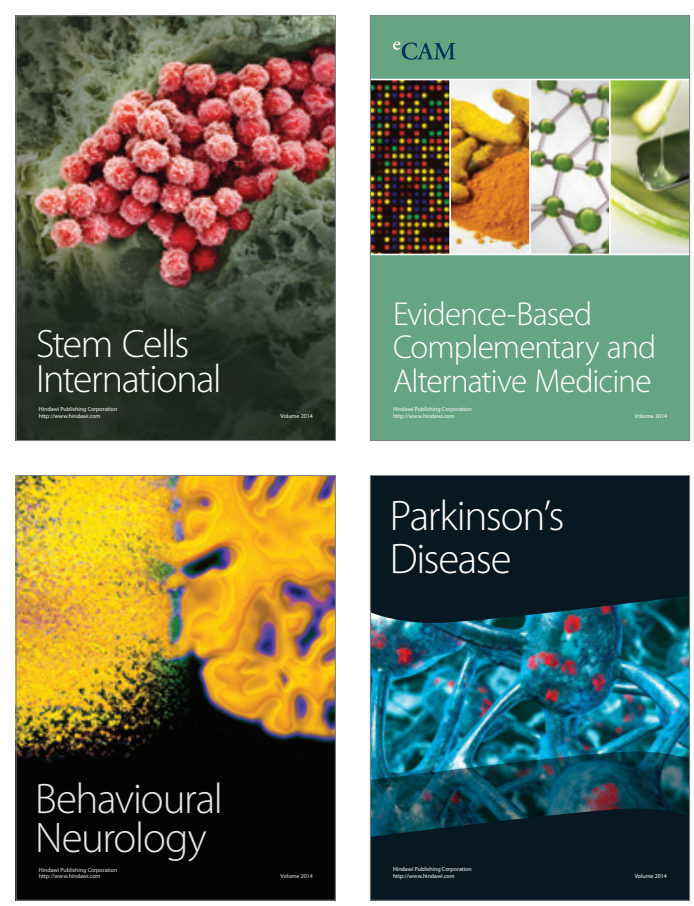

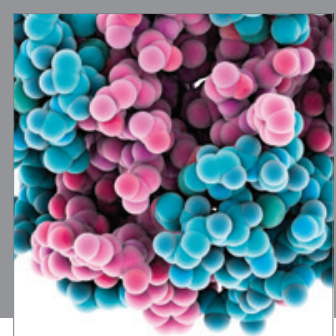

Journal of
Diabetes Research

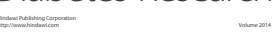

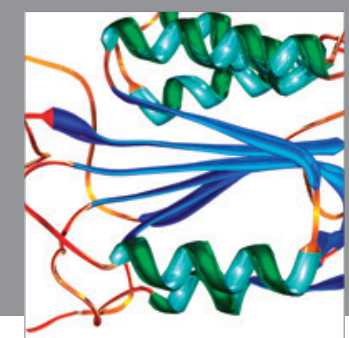

Disease Markers
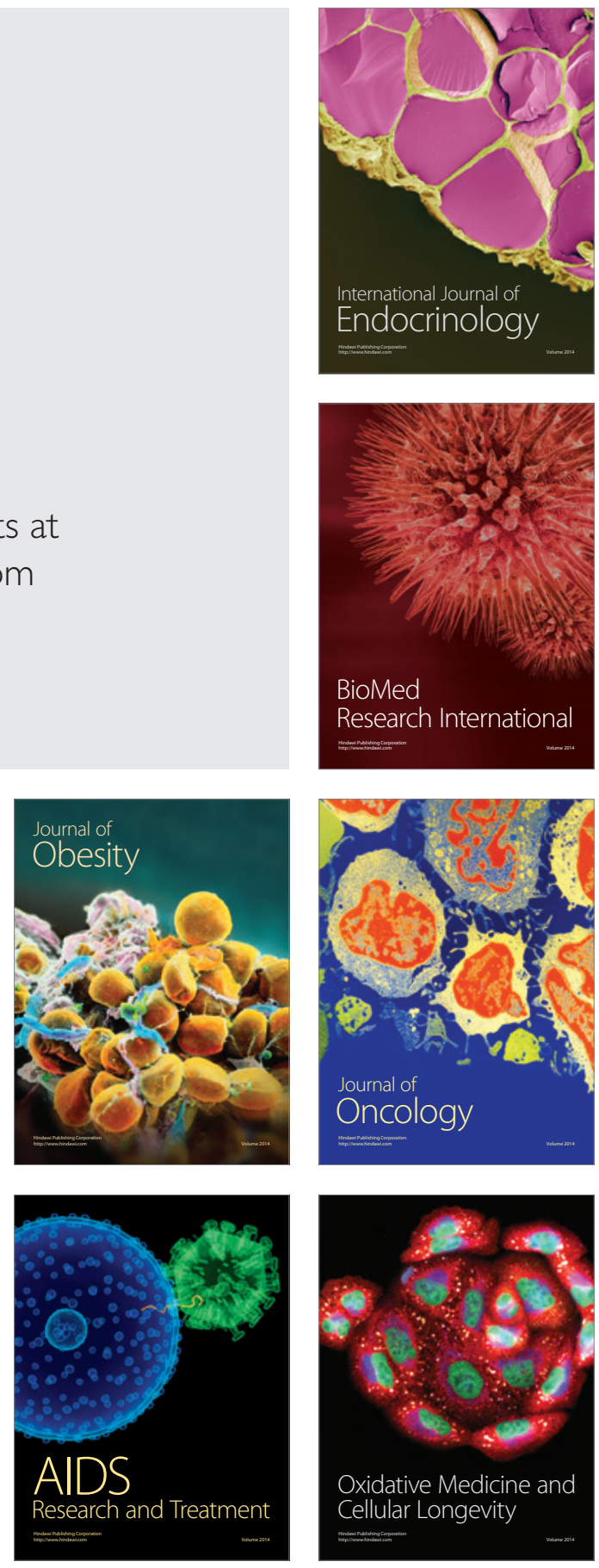